\title{
A knowledge-based system for materials selection in mechanical engineering design
}

\begin{abstract}
This paper studies various work on the development of computerized material selection system. The importance of knowledge-based system (KBS) in the context of concurrent engineering is explained. The study of KBS in material selection in an engineering design process is described. The development in materials databases, which sometimes serve as material selection packages, is also discussed. The use of KBS in material selection and the application in the domain of polymeric-based composite are chosen as typical examples.
\end{abstract}

Keyword: Material selection; Knowledge-based system; Materials database; Polymericbased composite; Concurrent engineering; Mechanical engineering design 